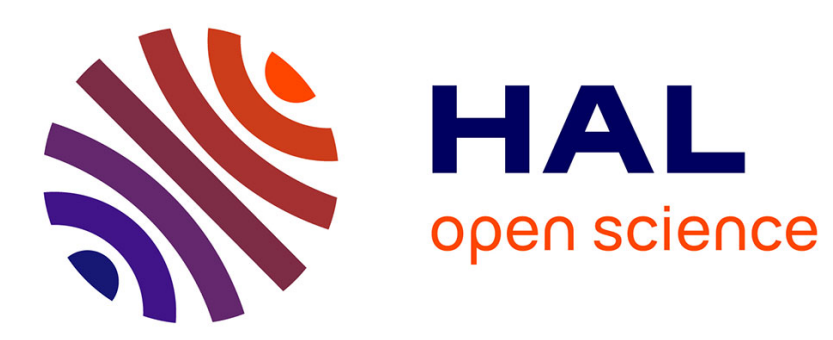

\title{
A monotonic algorithm for the optimal control of the Fokker-Planck equation
}

Guillaume Carlier, Julien Salomon

\section{To cite this version:}

Guillaume Carlier, Julien Salomon. A monotonic algorithm for the optimal control of the FokkerPlanck equation. Proceedings of the 47th IEEE Conference on Decision and Control., Dec 2008, Cancun, Mexico. hal-00267432

\section{HAL Id: hal-00267432 \\ https://hal.science/hal-00267432}

Submitted on 27 Mar 2008

HAL is a multi-disciplinary open access archive for the deposit and dissemination of scientific research documents, whether they are published or not. The documents may come from teaching and research institutions in France or abroad, or from public or private research centers.
L'archive ouverte pluridisciplinaire $\mathbf{H A L}$, est destinée au dépôt et à la diffusion de documents scientifiques de niveau recherche, publiés ou non, émanant des établissements d'enseignement et de recherche français ou étrangers, des laboratoires publics ou privés. 


\title{
A monotonic algorithm for the optimal control of the Fokker-Planck equation
}

\author{
Guillaume Carlier and Julien Salomon
}

\begin{abstract}
Motivated by some crowd motion models in the presence of noise, we consider an optimal control problem governed by the Fokker-Planck equation. We sketch optimality conditions by means of an Hamilton-Jacobi-Bellman equation and we give a monotonic scheme for the numerical approximation of the solution.
\end{abstract}

\section{INTRODUCTION}

Our aim is to solve numerically the following optimization problem:

$$
\begin{aligned}
\inf E(\rho, v):= & \int_{0}^{1} \int_{\mathbb{R}^{d}} \frac{1}{2}|v(t, x)|^{2} \rho(t, x) \mathrm{d} x \mathrm{~d} t \\
& +\int_{\mathbb{R}^{d}} V(x) \rho(1, x) \mathrm{d} x
\end{aligned}
$$

governed by the Fokker-Planck equation

$$
\partial_{t} \rho-\varepsilon^{2} \Delta \rho+\operatorname{div}(\rho v)=0 \text { on }(0,1) \times \mathbb{R}^{d}
$$

with (given) initial datum $\rho(0,)=.\rho_{0}$ where $\rho_{0}$ is a probability measure on $\mathbb{R}^{d}$.

The main motivation for this model comes from macroscopic crowd motion modelling (see the recent papers of Maury and Venel [10] and Buttazzo, Oudet and Jimenez [3]). Let us mention that in the case $\varepsilon=0$ and if one imposes a prescribed value $\rho_{1}$ to $\rho(1,$.$) instead of having a terminal$ cost in (1), then the minimal value of the cost is exactly the squared 2-Wasserstein distance between $\rho_{0}$ and $\rho_{1}$ : this is the dynamic formulation of the optimal transportation due to Brenier and Benamou [1]. In [3], Buttazzo, Jimenez and Oudet, building upon Brenier-Benamou formulation, consider the minimization of the sum of the kinetic energy and an additional term taking into account congestion effects (e.g. the integral of $\rho^{2}$ ), the state equation is the continuity equation $(\varepsilon=0)$ and both initial and terminal probabilities $\rho_{0}$ and $\rho_{1}$ are prescribed. In the model (1)-(2), one looks for the optimal motion of a crowd that is initially distributed according to $\rho_{0}$ and whose dynamics is governed by (2). As usual, the diffusion term in (2) amounts to considering that the individual dynamics of particles is governed by a stochastic differential equation with volatility $\sqrt{2} \varepsilon$. The cost functional $E$ that we consider involves the total kinetic energy (as in [1] and [3]) plus a terminal cost given by a potential $V$. The interpretation of this terminal cost is that

This work was supported by the french A.N.R, "Programme blanc OTARIE" and "Programme blanc C-Quid"

G. Carlier and J. Salomon, CEREMADE, UMR CNRS 7534, Université Paris IX Dauphine, Pl. de Lattre de Tassigny, 75775 Paris Cedex 16, France. carliercceremade.dauphine.fr, salomon@ceremade. dauphine.fr the crowd aims to reach zones of low potential $V$ at the terminal time 1 (as an extreme case, if $V$ is 0 on some set $K$ and $+\infty$ elsewhere then the aim of the crowd is to be in the safe zone $K$ at the terminal time). Some variants and generalizations of this simple model present some similarities with Mean-Field Games recently introduced by J.-M. Lasry and P.-L. Lions ([5] [6] [7]).

\section{OPTIMALITY CONDITIONS}

In this section, we give an informal description of the optimality conditions for the minimization problem (1)-(2) (existence results and detailed proofs will be given in [4]). Let us start by remarking, as in Brenier and Benamou [1] that $E$ is strictly convex in the variables $(\rho, m):=(\rho, \rho v)$ and the Fokker-Planck equation is linear in these variables, there is therefore uniqueness of the minimizer. Let us now note that (2) together with the Cauchy datum $\rho(0,)=.\rho_{0}$ can be written in the weak form as

$$
\begin{array}{r}
\int_{\mathbb{R}^{d}}\left(\phi(1, x) \rho(1, x)-\phi(0, x) \rho_{0}(x)\right) \mathrm{d} x \\
=\int_{0}^{1} \int_{\mathbb{R}^{d}}\left(\partial_{t} \phi+\varepsilon^{2} \Delta \phi+v \cdot \nabla \phi\right) \rho
\end{array}
$$

for every $\phi \in C_{c}^{\infty}\left(\mathbb{R} \times \mathbb{R}^{d}\right)$. Following Brenier and Benamou [1], we then introduce the Lagrangian:

$$
\begin{aligned}
\mathcal{L}(\rho, v, \phi):=E(\rho, v) & +\int_{0}^{1} \int_{\mathbb{R}^{d}}\left(\partial_{t} \phi+\varepsilon^{2} \Delta \phi+v \cdot \nabla \phi\right) \rho \\
& -\int_{\mathbb{R}^{d}} \phi(1, .) \rho(1, .)+\int_{\mathbb{R}^{d}} \phi(0, .) \rho_{0}
\end{aligned}
$$

and rewrite (1)-(2) as:

$$
\inf _{(\rho, v)} \sup _{\phi} \mathcal{L}(\rho, v, \phi)
$$

The (formal) dual is obtained by permuting the inf and the sup and the conditions characterizing the saddle-point of $\mathcal{L}$ read as (2) together with:

$$
\begin{array}{r}
v=-\nabla \phi \\
\partial_{t} \phi+\frac{1}{2} v^{2}+v \cdot \nabla \phi+\varepsilon^{2} \Delta \phi=0 \\
\phi(1, .)-V=0 .
\end{array}
$$

Thus, at least formally (i.e. ignoring regularity issues), the minimization problem (1)-(2) admits a unique solution $(\rho, v)$ where the optimal velocity is given by $v:=-\nabla \phi$ and $\phi$ 
is the solution of the (backward) Hamilton-Jacobi-Bellman equation:

$$
\begin{aligned}
\partial_{t} \phi+\varepsilon^{2} \Delta \phi-\frac{1}{2}|\nabla \phi|^{2} & =0, \\
\phi(1, .) & =V .
\end{aligned}
$$

Now, the optimal density $\rho$ is obtained by solving the FokkerPlanck equation (2) with $v=-\nabla \phi$, which can be done as follows. Considering the stochastic differential equation (assuming $\phi$ is regular enough):

$$
d X_{t}^{x}=-\nabla \phi\left(t, X_{t}\right) \mathrm{d} t+\sqrt{2} \varepsilon \mathrm{d} B_{t}, X_{0}^{x}=x
$$

(where $\left(B_{t}\right)_{t}$ is the standard Brownian motion) then it is a well-known consequence of Itô's lemma that $\rho(t,$.$) is the$ probability law of $X_{t}$ whenever $X_{0}$ is distributed according to the probability $\rho_{0}$, which more precisely means

$$
\int_{\mathbb{R}^{d}} \psi(x) \rho(t, x) \mathrm{d} x=\int_{\mathbb{R}^{d}} \mathbb{E}\left(\psi\left(X_{t}^{x}\right)\right) \rho_{0}(x) \mathrm{d} x
$$

for every test-function $\psi$. The description above deals with the problem (1)-(2) on the whole space. In our numerical simulations, we will focus on the case of a bounded domain $\Omega$ (which requires to consider additional boundary conditions like the Neumann homogeneous boundary conditions $v \cdot n=$ 0 in (2) and $\partial_{n} \phi=0$ in (8) on $\left.(0,1) \times \partial \Omega\right)$.

\section{Discrete SETTING}

Before presenting the optimization algorithm, we introduce the following discretization of the problem. Even if what follows easily generalizes to $2 \mathrm{D}$ and $3 \mathrm{D}$ situations, we will focus in the rest of that paper on the $1 D$-case for the sake of simplicity. Given a matrix $C$, we denote by $C^{*}$ its transpose matrix.

Let us consider two positive integers $M, N$ and a positive real number $L$. We consider here the case of the bounded space domain $[0, L]$ for (2). We define the time and space steps by $d t=\frac{1}{N}$ and $d x=\frac{L}{M}$ and denote for $j=0, \ldots, M$, $i=0, \ldots, N$ by $\rho_{j}^{i}$ and $\rho_{j}$ the numerical approximations of $\rho(i . d t, j . d x)$ and $\rho(\cdot, j . d x)$ respectively. For reasons that will appear later, the discrete velocity is defined at the points $(i . d t,(j+1 / 2) . d x)$, and is therefore denoted it by $v_{j+1 / 2}^{i}$. To be consistent with the fact that the total mass of $[0, L]$ is constant in time, at the discrete level, we impose the homogeneous Neumann boundary condition that is expressed by $v_{1 / 2}^{i}=v_{M-1 / 2}^{i}=0$, for $i=0 \ldots N-1$. We shall also use the notations $v^{i}=\left(v_{j+1 / 2}^{i}\right)_{j=1 \ldots M-1}$ and $\rho^{i}=$ $\left(\rho_{j}^{i}\right)_{j=1 \ldots M-1}$.

\section{A. Discretized cost functional}

From now on, $v$ stands for the discrete velocity $\left(v_{j+1 / 2}^{i}\right)_{i, j}$ and $V$ is the vector of components $V_{j}=V(j . d x)$. We consider the following discrete version of the cost functional E:

$$
\begin{aligned}
E_{d t, d x}(v): & =d t . d x \sum_{i=0}^{N-1} \sum_{j=1}^{M-1} \frac{1}{2} q_{j}\left(v^{i}\right) \rho_{j}^{i}+d x \sum_{j=1}^{M-1} V_{j} \rho_{j}^{N} \\
& =d t \sum_{i=0}^{N-1} \frac{1}{2}\left\langle\rho^{i}, q\left(v^{i}\right)\right\rangle+\left\langle\rho^{N}, V\right\rangle
\end{aligned}
$$

where $\langle\cdot, \cdot\rangle$ is the scalar product on $\mathbb{R}^{M-1}$ defined by:

$$
\langle u, v\rangle=d x \sum_{j=1}^{M-1} u_{j} v_{j}
$$

The vector $q\left(v^{i}\right)=\left(q_{j}\left(v^{i}\right)\right)_{j=1 \ldots M-1}$ is defined from $v^{i}$ by:

$$
q_{j}\left(v^{i}\right)=\frac{\left(v_{j-1 / 2}^{i}\right)^{2}+\left(v_{j+1 / 2}^{i}\right)^{2}}{2} .
$$

This choice corresponds to use a trapezoid rule to approximate $|v|^{2}$ in the first integral of (1).

\section{B. Numerical scheme for the Fokker-Planck equation}

The preservation of the positivity of $\rho$ at the discrete level appears in numerical simulation as a crucial issue, especially for small values of $\varepsilon$. Indeed, the linearity of $E$ with respect to $\rho$ leads to numerical instabilities when using schemes which do not possess this property, independently of their order of accuracy. This fact motivates the use of a low order Godunov scheme for the advective part of (2) which ensures both small computational cost and positivity of the numerical solutions.

Remark 1: In addition, this framework enables us to avoid the introduction of Lagrange multipliers corresponding to the constraint $\rho \geq 0$.

We are now in the position to define the numerical solver of (2). Starting from $\rho_{j}^{0}=\rho_{0}(j . d x), \rho_{j}^{i}$ is computed iteratively for $j=1 \ldots M-1$ by:

$$
\begin{aligned}
\rho_{j}^{i+1}= & \rho_{j}^{i}+\varepsilon^{2} \frac{d t}{d x^{2}}\left(\rho_{j+1}^{i}-2 \rho_{j}^{i}+\rho_{j-1}^{i}\right) \\
& -\frac{d t}{d x}\left(\rho_{j+1 / 2}^{i} v_{j+1 / 2}^{i}-\rho_{j-1 / 2}^{i} v_{j-1 / 2}^{i}\right) .
\end{aligned}
$$

In this equation, the terms $\rho_{j+1 / 2}^{i}$ and $\rho_{j-1 / 2}^{i}$ of the advective part are defined according to a Godunov scheme, i.e. using up-winding:

$$
\rho_{j+1 / 2}^{i}=\left\{\begin{array}{cll}
\rho_{j+1}^{i} & \text { if } & v_{j+1 / 2}^{i}<0 \\
\rho_{j}^{i} & \text { if } & v_{j+1 / 2}^{i} \geq 0 .
\end{array}\right.
$$

To simplify our notations, we rewrite (11) as:

$$
\rho^{i+1}=\left(A+B\left(v^{i}\right)\right) \rho^{i}
$$

where $A$ corresponds to the identity matrix plus the discrete Laplace operator, i.e. the first two terms of the right handside of (11), and $B$ is associated to the advective part, i.e. the last term of the right hand-side of (11).

Given a positive vector $\rho^{i}$, it is a simple matter to check that a sufficient condition to guarantee the positivity of $\rho^{i+1}$ (and the stability of the scheme) is:

$$
\forall j=1 \ldots M-1,\left|v_{j+1 / 2}^{i}\right| \leq \lambda:=\frac{d x}{2 d t}-\varepsilon^{2} \frac{1}{d x} .
$$




\section{OPTIMIZATION PROCEDURE}

In order to compute efficiently a minimizing sequence, we use a so called monotonic scheme. This approach has already shown a great efficiency in the field of optimal quantum control [12], [8], [9], where gradient methods usually lead to numerical instabilities. These types of algorithms apply for bilinear control problems and are based on a special factorization of the variations in $E_{d t, d x}$ that is presented in the next sections.

\section{A. Adjoint state}

In the approach we follow, a crucial role is played by the adjoint state $\phi^{i}=\left(\phi_{j}^{i}\right)_{j=1 \ldots M-1}$ that is defined iteratively for $i=0 \ldots N$, by the backward propagation:

$$
\begin{aligned}
\phi^{N} & =V, \\
\phi^{i} & =\left(A^{*}+B^{*}\left(v^{i}\right)\right) \phi^{i+1}+\frac{d t}{2} q\left(v^{i}\right) .
\end{aligned}
$$

This variable is the discrete version of the Lagrange multiplier defined by (5-7).

\section{B. Variations in $E_{d t, d x}$}

We present now the algebraic manipulations at the heart of the monotonic schemes. Let us consider two controls $v$ and $v^{\prime}$ and the corresponding solutions $\left(\rho^{i}\right)_{i=0 \ldots N}$ and $\left(\rho_{i}^{\prime}\right)_{i=0 \ldots N}$ of (11). In what follows, the adjoint $\phi=\left(\phi_{i}\right)_{i=0 \ldots N}$ corresponds to $v$. One has:

$$
\begin{array}{r}
E_{d t, d x}\left(v^{\prime}\right)-E_{d t, d x}(v)=\frac{d t}{2} \sum_{i=0}^{N-1}\left\langle\rho^{\prime i}, q\left(v^{i}\right)-q\left(v^{i}\right)\right\rangle \\
+\frac{d t}{2} \sum_{i=0}^{N-1}\left\langle\rho^{\prime i}-\rho^{i}, q\left(v^{i}\right)\right\rangle \\
+\sum_{i=0}^{N-1}\left\langle\rho^{i+1}-\rho^{i+1}, \phi^{i+1}\right\rangle-\left\langle\rho^{i}-\rho^{i}, \phi^{i}\right\rangle \\
=\frac{d t}{2} \sum_{i=0}^{N-1}\left\langle\rho^{\prime i}, q\left(v^{i}\right)-q\left(v^{i}\right)\right\rangle \\
+\sum_{i=0}^{N-1}\left\langle\left(B\left(v^{i}\right)-B\left(v^{i}\right)\right) \rho^{\prime i}, \phi^{i+1}\right\rangle .
\end{array}
$$

This identity can also be expressed locally through the formula:

$$
E_{d t, d x}\left(v^{\prime}\right)-E_{d t, d x}(v)=d t . d x \sum_{i=0}^{N-1} \sum_{j=1}^{M-2} \Delta_{j}^{i}\left(v^{\prime}, v\right),
$$

where:

$$
\begin{array}{r}
\Delta_{j}^{i}\left(v^{\prime}, v\right)=\frac{\rho_{j}^{i}+\rho_{j+1}^{i}}{2}\left(\frac{\left(v_{j+1 / 2}^{i}\right)^{2}-\left(v_{j+1 / 2}^{i}\right)^{2}}{2}\right) \\
+\left(\rho_{j+1 / 2}^{i} v_{j+1 / 2}^{\prime i}-{\widetilde{\rho^{\prime}}}_{j+1 / 2}^{i} v_{j+1 / 2}^{i}\right)\left(\frac{\phi_{j+1}^{i+1}-\phi_{j}^{i+1}}{d x}\right) .
\end{array}
$$

In this equation, we have introduced

$$
{\widetilde{\rho^{\prime}}}^{i}{ }_{j+1 / 2}^{i}=\left\{\begin{array}{ccc}
\rho_{j+1}^{i} & \text { if } \quad v_{j+1 / 2}^{i}<0 \\
\rho_{j}^{\prime i} & \text { if } & v_{j+1 / 2}^{i} \geq 0
\end{array}\right.
$$

Remark 2: Given $v$, note that the value of $\rho_{j+1 / 2}^{i}$ depends on the sign of $v_{j}^{\prime i}$, so that $v_{j}^{\prime i} \mapsto \Delta_{j}^{i}\left(v^{\prime}, v\right)$ is a continuous, piecewise polynomial function.

\section{Optimization strategy}

This section provides a brief exposition of the optimization strategy we follow to solve our problem.

Given $v$ and a positive real number $\theta$, We define $v_{j}^{\prime i}$ as a solution of

$$
\Delta_{j}^{i}\left(v^{\prime}, v\right)=-\theta \frac{\rho_{j}^{\prime i}+\rho_{j+1}^{\prime i}}{2}\left(v_{j}^{i}-v_{j}^{i}\right)^{2} .
$$

According to Remark 2, this equation may have one, two or four roots, including the trivial one $v_{j}^{\prime i}=v_{j}^{i}$. When possible, we define $v_{j}^{\prime i}$ as the root of (15) that is closer to $v_{j}^{i}$, and we set $v_{j}^{\prime i}=v_{j}^{i}$ otherwise. Thus, the monotonicity of our algorithm is guaranteed.

Let us give the explicit formula corresponding to this procedure. We suppose that $\frac{\rho_{j}^{\prime i}+\rho_{j+1}^{\prime i}}{2}>0$, otherwise the contribution of this term is zero for all choice of $v_{j+1 / 2}^{\prime i}$. We introduce

$$
\begin{aligned}
\delta & =\frac{2}{\theta+1}, \\
\nu_{j+1 / 2}^{i} & =\frac{2 \rho_{j+1 / 2}^{i}}{\rho_{j}^{i}+\rho_{j+1}^{\prime i}},
\end{aligned}
$$

and

$$
\widetilde{\nu}_{j+1 / 2}^{i}=\frac{2{\widetilde{\rho^{\prime}}}_{j+1 / 2}^{i}}{\rho_{j}^{i i}+\rho_{j+1}^{\prime i}}
$$

Consider now:

$$
\begin{aligned}
& \alpha_{j+1 / 2}^{i}=(1-\delta) v_{j+1 / 2}^{i}+\delta \nu_{j+1 / 2}^{i} \frac{\phi_{j+1}^{i+1}-\phi_{j}^{i+1}}{d x}, \\
& \beta_{j+1 / 2}^{i}= \\
& \frac{-b_{j+1 / 2}^{i}-\operatorname{sign}\left(v_{j+1 / 2}^{i}\right) \sqrt{\left(b_{j+1 / 2}^{i}\right)^{2}-4 a_{j+1 / 2}^{i} \cdot c_{j+1 / 2}^{i}}}{2 a_{j+1 / 2}^{i}},
\end{aligned}
$$

where sign is the function

$$
\operatorname{sign}(x)=\left\{\begin{array}{cll}
1 & \text { if } & x \geq 0 \\
-1 & \text { if } & x<0
\end{array}\right.
$$

and :

$$
\begin{aligned}
a_{j+1 / 2}^{i} & =1+\theta, \\
b_{j+1 / 2}^{i} & =-2\left(\theta v_{j+1 / 2}^{i}+\nu_{j+1 / 2}^{i} \frac{\phi_{j+1}^{i+1}-\phi_{j}^{i+1}}{d x}\right), \\
c_{j+1 / 2}^{i} & =\theta\left(v_{j+1 / 2}^{i}\right)^{2}+\widetilde{\nu}_{j+1 / 2}^{i} \frac{\phi_{j+1}^{i+1}-\phi_{j}^{i+1}}{d x} .
\end{aligned}
$$

The control $v_{j}^{\prime i}$ can be expressed by :

$$
v_{j+1 / 2}^{i}=\left\{\begin{array}{lll}
\alpha_{j+1 / 2}^{i} & \text { if } & v_{j+1 / 2}^{i} \cdot \alpha_{j+1 / 2}^{i} \geq 0 \\
\beta_{j+1 / 2}^{i} & \text { if } & v_{j+1 / 2}^{i} \cdot \alpha_{j+1 / 2}^{i}<0 .
\end{array}\right.
$$




\section{Constraints on $v$}

We recall that the bound (12) on the velocity $v$ is crucial for the positivity of the density. As the stability of our optimization scheme is based on the positivity of the variable $\rho$, the strategy presented in the last section has to include a slop-limiter such that (12) prevails. Instead of defining $v_{j+1 / 2}^{i i}$ through (16), we alternatively denote by $\check{v}_{j+1 / 2}^{i}$ the value obtained in (16) and consider the definition:

$$
v_{j+1 / 2}^{\prime i}=\operatorname{sign}\left(\check{v}_{j+1 / 2}^{i}\right) \cdot \min \left(\lambda,\left|\check{v}_{j+1 / 2}^{i}\right|\right) .
$$

It is easy to check that this modification does not spoil the monotonicity of our procedure, as soon as $v$ satisfies (12).

\section{E. Algorithm}

We can now define precisely our optimization algorithm. Suppose that $v^{k}$ is given. The computation of $v^{k+1}$ is achieved as follows.

- Define $\phi^{k}$ by (13) with $v=v^{k}$.

- Define $\rho^{0}=\rho_{0}$ and compute iteratively $\rho^{i}$ from $\rho^{i-1}$ according to the sub-steps:

- define $\left(v^{k+1}\right)^{i-1}$ by (17) where $\check{v}_{j+1 / 2}^{i}$ is computed with $\phi=\phi^{k}$

- define $\left(\rho^{k+1}\right)^{i}$ by (11) with $v^{i-1}=\left(v^{k+1}\right)^{i}$.

A possible termination criterion is obtained by checking the discrete optimality conditions, i.e., given a tolerance threshold Tol $>0$ :

$$
\begin{aligned}
\sup _{1 \leq i \leq N-1,1 \leq j \leq M-1} & \left(\mid \frac{\left(\rho^{k}\right)_{j}^{i}+\left(\rho^{k}\right)_{j+1}^{i}}{2}\left(v^{k}\right)_{j+1 / 2}^{i}\right. \\
& \left.+\left(\rho^{k}\right)_{j+1 / 2}^{i} \frac{\left(\phi^{k}\right)_{j+1}^{i+1}-\left(\phi^{k}\right)_{j}^{i+1}}{d x} \mid\right) \leq \text { Tol. }
\end{aligned}
$$

Remark 3: Note that the necessity of the computation of $\rho$ only comes from the Godunov scheme. Indeed, the formal gradient of the Lagrangian with respect to $v$ is:

$$
\nabla_{v} \mathcal{L}(\rho, v, \phi)=\rho(v+\nabla \phi),
$$

so that only $v$ and $\phi$ are necessary to determine the descent direction. This additional computation substitutes the computation of a Lagrange multiplier associated to the positivity constraint that applies on $\rho$.

\section{NUMERICAL RESULTS}

This section aims at presenting some numerical results obtained with our approach. In our algorithm we choose $\theta=1$.

Numerical simulations exhibit very good convergence results. This is explained by the fact that the discrete energy $E_{d t, d x}$ decreases with the iterations of our algorithm and by the strict convexity of $E$ in the variables $(\rho, \rho v)$ which yields uniqueness of the minimizer characterized by the conditions of section II.

\section{A. The 1D-case}

We first focus on the computation of optimal transportation between two Gaussian densities in $\Omega=[0,1]$. We choose:

$$
V(x)=5\left(1-e^{-10(x-0.2) .^{2}}-e^{-10(x-0.8)^{2}}\right) .
$$

In a first example, the initial probability is symmetric with respect to space and defined by :

$$
\rho(x, 0)=e^{-10(x-0.5)^{2}} .
$$

A second test is done with a initial condition that has been slightly shifted to the right. These two results are obtained with $\varepsilon=0$. The case $\varepsilon>0$ is treated in a third test.

These functions and the final state are represented on Fig. 1. A fast convergence is observed. In the framework we work,
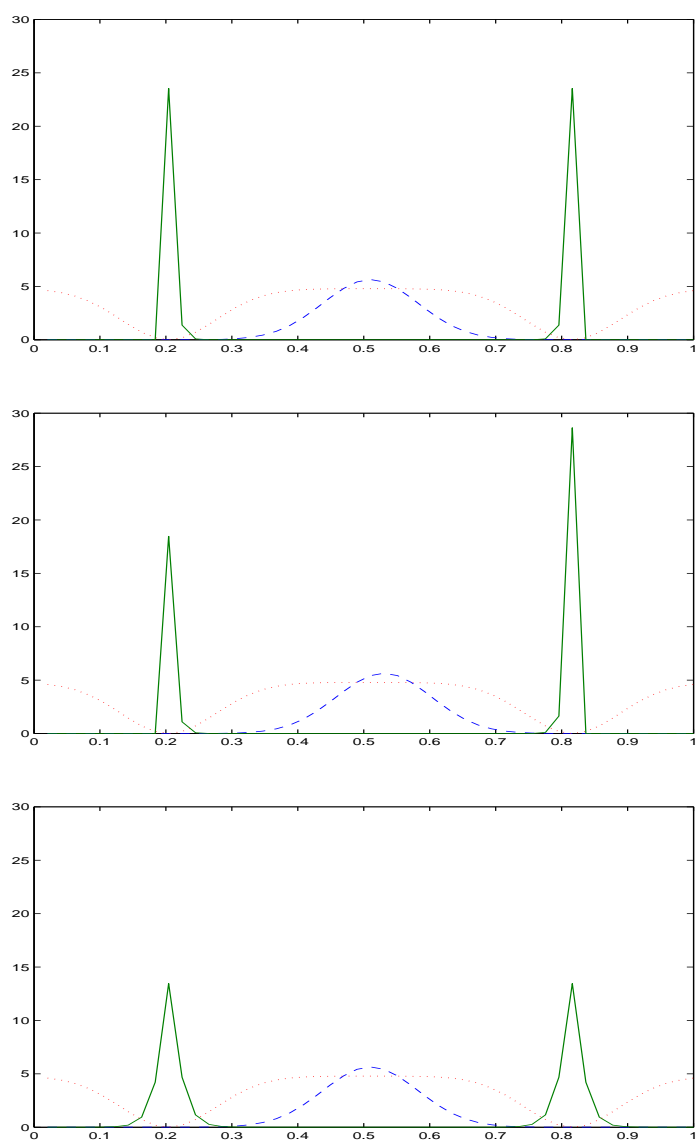

Fig. 1. Evolution of $\rho$ in the $1 D$-case. Solid line : initial datum, dashed line : final probability, dotted line : potential. Top: symmetric initial probability. Middle: slightly shifted initial probability. Bottom: case $\varepsilon^{2}=1 O^{-2}$.

the control $v$ has $5.10^{4}$ components. Only 100 iterations, and a CPU time ${ }^{1}$ of $196.64 \mathrm{~s}$ are required to obtained numerical convergence. The cost functional values together with the termination criterion defined in (18) are depicted on Fig. 2.

\footnotetext{
${ }^{1}$ The computation are done using the free software OCTAVE (http://www.octave.org ).
} 


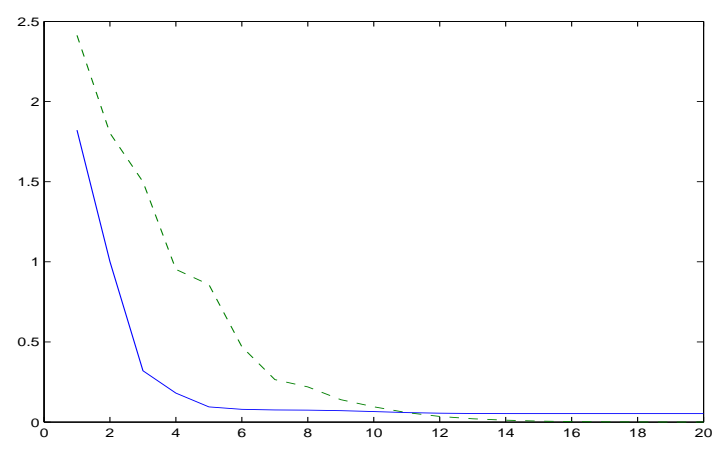

Fig. 2. Numerical convergence in the 20 first iterations. Solid line : Values of $E_{d t, d x}$. dashed line : values of the termination criterion defined in (18) (rescaled).

\section{B. The $2 D$ - case}

The algorithm is tested in the 2-dimensional case, with $\Omega=[0,1] \times[0,1]$. We consider the initial probability :

$$
\rho(x, y, 0)=e^{-10(x-0.2)^{2}}+e^{-10(y-0.2)^{2}},
$$

and the potential :

$$
\begin{array}{r}
V(x, y)=40\left(1+e^{-10(y-0.8)^{2}}-e^{-10(x-0.8)^{2}}\right. \\
\left.+e^{-10(y-0.5)^{2}}-e^{-10(x-0.5)^{2}}\right) .
\end{array}
$$

This potential is represented on Fig. 3. The evolution of the

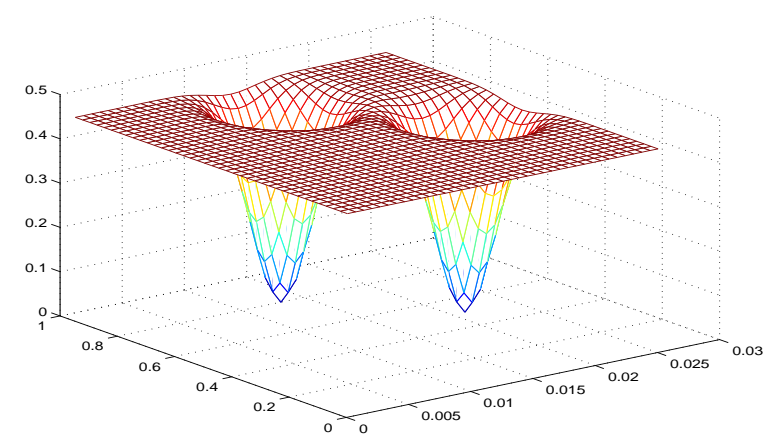

Fig. 3. Potential $V(x, y)$.

probability $\rho$ during the transportation is depicted on Fig. 4. As it was the case in $1 D$, the numerical convergence is obtained in about 100 iterations.

\section{ACKNOWLEDGMENTS}

J.S. gratefully acknowledges helpful discussions on this topic with F. Lagoutière (Laboratoire J-L. Lions, Université Paris VII).

\section{REFERENCES}

[1] J.-D. Benamou and Y. Brenier, A computational fluid mechanics solution to the Monge-Kantorovich mass transfer problem , Numer. Math., vol. 84 (3), 2000, pp 375-393.
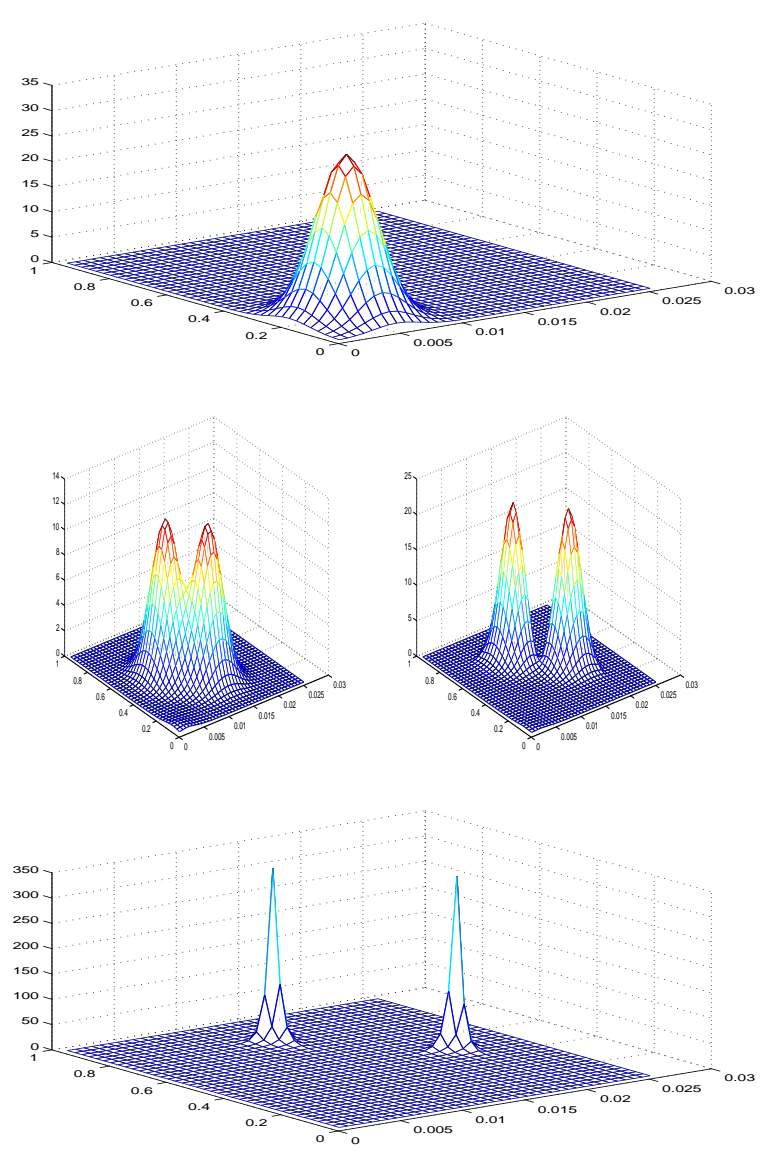

Fig. 4. Evolution of $\rho$ in the $2 D$-case. Top: initial probability. Middle: probability at time $t=.5$ and $t=.75$. Bottom: final probability.

[2] Y. Brenier, Polar factorization and monotone rearrangement of vectorvalued functions, Comm. Pure Appl. Math., vol. 44 (4), 1991, pp 375417.

[3] G. Buttazzo, E. Oudet and C. Jimenez, An optimization problem for mass transportation with congested dynamics, preprint available at http://cvgmt.sns.it/people/buttazzo/, 2007.

[4] G. Carlier and J. Salomon, work in preparation.

[5] J.-M. Lasry and P.-L. Lions, Jeux à champ moyen. I. Le cas stationnaire. (French) [Mean field games. I. The stationary case], C. R. Math. Acad. Sci. Paris, vol. 343 (9), 2006, pp 619-625.

[6] J.-M. Lasry and P.-L. Lions, Jeux à champ moyen. II. Horizon fini et contrôle optimal. (French) [Mean field games. II. Finite horizon and optimal control], C. R. Math. Acad. Sci. Paris, vol. 343 (10), 2006, pp 679-684.

[7] J.-M. Lasry and P.-L. Lions, Mean field games, Jpn. J. Math., vol. 2 (1), 2007, pp 229-260.

[8] Y. Maday and G. Turinici, New formulations of monotonically convergent quantum control algorithms, J. Chem. Phys., vol. 118 (18), 2003, pp 8191-8196.

[9] Y. Maday, J. Salomon and G. Turinici, Monotonic time-discretized schemes in quantum control, Num. Math, vol. 103 (2), 2006, pp 323338.

[10] B. Maury and J. Venel, Un modèle de mouvements de foule (in french), ESAIM Proceedings, vol. 18, 2007, pp 143-152.

[11] C. Villani, Topics in optimal transportation, Graduate Studies in Mathematics, 58, American Mathematical Society, Providence, RI; 2003.

[12] W. Zhu and H. Rabitz, A rapid monotonically convergent iteration algorithm for quantum optimal control over the expectation value of a positive definite operator, J. Chem. Phys., vol. 109, 1998, pp 385-391. 